\section{Incidencia y letalidad intrahospitalaria por insuficiencia cardiaca en Chile: ¿Existen diferencias por sexo?}

FELIPE DÍAZ-TORO ${ }^{1, a, b}$, CAROLINA NAZZAL N. 2,b,c , HUGO VERDEJO P., ${ }^{3, c}$

\section{Incidence and hospital mortality due to heart failure. Are there any differences by sex?}

Background: Gender may influence the incidence, severity and hospital mortality due to heart failure (HF). Aim: To evaluate the influence of sex on the proportion of patients hospitalized due to heart failure, its incidence and hospital mortality. Methods: Analysis of the hospital discharge database of the Chilean Ministry of Health during 2014. All hospital admissions for HF were considered according to ICD-10 codes, including the discharge diagnosis of congestive HF (I500), left ventricular HF (I501) and non-specified HF (I509). Incidence rates, proportion of discharges due to HF and hospital mortality were calculated according to age and sex. Results: During 2014, there were 1,306,431 discharges from Chilean hospitals. Of these, 125,484 were for cardiovascular disease and 10\% of these corresponded to HF (12,825). The incidence rate was slightly higher in men than in women (0.71 and 0.70 per 1,000 admissions respectively). Among patients aged 80 years or more, the prevalence of admissions for HF was higher in women (19.1 and $15.9 \%$ respectively, $p<0.01$ ). Hospital mortality was also higher in women (9.7 and $8.6 \%$ respectively, $p=0.03$ ). The factors associated with a higher hospital mortality were an age over 80 years (Odds Ratio (OR) 2.11; 95\% confidence intervals $(C I): 1.87-2.40 ; p<0.01)$, a length of stay over seven days (OR 1.13; 95\%CI: 1.01-1.29; $p=0.04)$, being admitted to high complexity facilities (OR 1.29; 95\%CI: 1.12-1.50; $p=0.01$ ) and being insured by the public national health fund (OR 1.94; 95\%CI: 1.54-2.43; $p<0.01$ ). Conclusions: The incidence of hospital admissions due to HF is similar in men and women. There is high hospital mortality, especially in women.

(Rev Med Chile 2017; 145: 703-709)

Key words: Chile; Heart Failure; Hospital Mortality; Incidence; Prevalence.

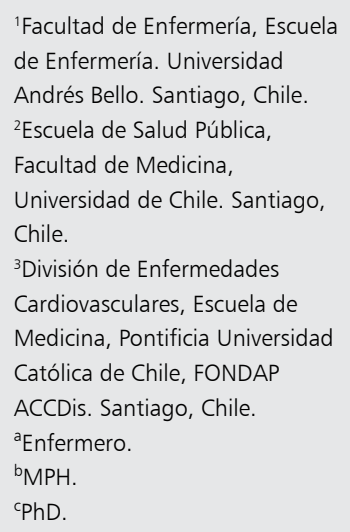

Los autores no declaran conflicto de intereses.

Trabajo no cuenta con

financiamiento.

Recibido el 16 de marzo de 2017 , aceptado el 27 de junio de 2017.

Correspondencia a:

Carolina Nazzal Nazal

Escuela de Salud Pública, Facultad de Medicina. Universidad de

Chile.

Avenida Independencia 939, Independencia. Santiago, Chile. cnazzal@med.uchile.cl

\section{L} a insuficiencia cardiaca (IC) representa un problema creciente de salud pública en el mundo: es la primera causa de hospitalización en población adulta ${ }^{1}$ y constituye una de las principales causas de morbimortalidad cardiovascular en los países desarrollados. Durante las últimas décadas, la prevalencia de IC ha aumentado en relación al envejecimiento de la población, al aumento en la sobrevida a los eventos coronarios y a la mejoría de los tratamientos farmacológicos de la enfermedad ${ }^{1}$. En registros internacionales, la prevalencia alcanza $3 \%$ en la población general y $8 \%$ en los mayores de 75 años ${ }^{1,2}$, y se espera que aumente a $4,5 \%$ al año $2030^{3}$.

A pesar de los importantes avances en su diagnóstico y tratamiento, la mortalidad por IC sigue siendo elevada. La letalidad intrahospitalaria fluctúa entre $2 \%$ y $20 \%{ }^{4}$, estimándose una cifra cercana a $11,5 \%$ en América Latina ${ }^{5,6}$, mientras que la sobrevida a cinco años es cercana a $50 \%$ una vez diagnosticada ${ }^{7}$.

En relación a la evolución y pronóstico de la 
enfermedad se han descrito diferencias por sexo, por ejemplo, en Estados Unidos de Norteamérica $52,6 \%$ de los casos prevalentes y $52,2 \%$ de los casos incidentes corresponden a mujeres, además 51\% de las hospitalizaciones y $57,8 \%$ de las muertes por IC ocurren en ellas ${ }^{8}$. Las mujeres con diagnóstico de IC son de mayor edad, con fracción de eyección preservada, mayor número de comorbilidades, mayor limitación funcional y peor calidad de vida que los hombres ${ }^{9,10}$. Pese a estas diferencias, en la mayoría de los estudios, el sexo no se asocia con mayor mortalidad intrahospitalaria ${ }^{11-15}$.

En Chile, los datos de morbimortalidad asociada a IC son prácticamente inexistentes. Este trabajo representa un primer intento de aproximación a las estadísticas vitales de IC mediante datos actuariales. Nuestro objetivo fue estimar las diferencias por sexo en la proporción, incidencia y letalidad de las hospitalizaciones por IC durante el año 2014; adicionalmente, se evaluaron los factores asociados a letalidad intrahospitalaria.

\section{Material y Métodos}

Estudio de corte transversal. Se consideraron todas las hospitalizaciones registradas con diagnóstico primario de IC en Chile durante el año 2014, en pacientes de 20 años y más, con los códigos 1500 (insuficiencia cardiaca congestiva), I501 (insuficiencia ventricular izquierda) e I509 (insuficiencia cardiaca no especificada), según la CIE 10. La información se obtuvo de la base de egresos hospitalarios del Departamento de Estadísticas e Información en Salud del Ministerio de Salud chileno (DEIS). En Chile, las cifras de egresos hospitalarios se obtienen a partir del Informe Estadístico de Egreso Hospitalario (Decreto No 1671/2010), cuyo reporte es obligatorio para todos los establecimientos de salud del territorio nacional, tanto públicos como privados. La base de datos generada incluye las siguientes variables: edad, sexo, previsión, comuna de egreso, región y servicio de salud, tipo de establecimiento, modalidad de atención, fecha de egreso, días de estadía y condición de egreso.

\section{Variables del estudio}

La condición de egreso se codifica como fallecido o vivo. Para este análisis, la variable previsión se categorizó según los pacientes pertenecieran al seguro público (FONASA) o privado y atención cerrada (ISAPRE, fuerzas armadas). Se excluyeron las hospitalizaciones sin información de previsión. A su vez, FONASA fue categorizada en dos tramos para diferenciar a los pacientes de menor nivel socioeconómico: A-B (beneficiarios carentes de recursos para cotizar en salud, condición de indigencia o con ingresos menores a USD 400 mensuales, aproximadamente) y C-D (incluye aquellos beneficiarios con ingresos mayor al límite anterior).

El tipo de establecimiento de egreso fue agrupado en dos categorías: centros de alta complejidad (dotación mayor a 300 camas, residencia médica y acceso a todas las especialidades médicas) y centros de baja complejidad (caracterizados por poseer un número menor a 300 de camas, no contar con médico las 24 h y número limitado de especialidades o solo medicina general). El resto de las variables se trataron en forma dicotómica o continua, según su naturaleza.

\section{Análisis estadístico}

Se describen las características sociodemográficas (sexo, edad, previsión) y asociadas a la hospitalización (días de estadía, tipo de establecimiento y condición de egreso) con mediana y percentiles 25-75 para las variables continuas y porcentajes para las categóricas.

Se calculó la tasa de incidencia de egresos por IC en población general por 1.000 habitantes. Para el cálculo de tasas, se estratificó la población por sexo y tramos de edad (20-44 años, 45-64 años, 6579 años y mayor o igual a 80 años). El numerador de la tasa está constituido por el número de egresos por IC y el denominador por la población adulta estimada en las proyecciones de población 20132020 para cada categoría estudiada (según cifras del Instituto Nacional de Estadísticas de Chile).

Para la estimación de prevalencias se calcularon las siguientes proporciones para cada grupo estratificado por edad y sexo: a) egresos hospitalarios ocurridos por IC del total de hospitalizaciones registradas en pacientes de 20 años y más $\mathrm{x} 100 \mathrm{y}$ b) egresos hospitalarios ocurridos por IC del total de hospitalizaciones cardiovasculares registradas en pacientes de 20 años y más x 100 (en este último caso el denominador incluye las hospitalizaciones registradas bajo los códigos I00-I99, CIE-10).

La letalidad intrahospitalaria por IC se estimó según la proporción de casos egresados fallecidos 
del total de casos de IC registrados (número de egresados fallecidos con diagnóstico de IC/ número total de egresos por IC en pacientes de 20 años y más x 100).

Se compararon las características entre hombres y mujeres mediante $\mathrm{t}$ de Student para las variables continuas y test de $\chi^{2}$ para las categóricas. Mediante regresión logística multivariada se evaluaron los factores asociados con letalidad intrahospitalaria; se calcularon los odds ratio (OR) e intervalos de confianza de 95\%. El nivel de significación considerado para todos los análisis fue $5 \%$ a dos colas y los análisis estadísticos fueron realizados con el software STATA versión 13.

\section{Resultados}

Se registraron 1.306.431 egresos en pacientes de 20 y más años durante el año 2014. De estos, $9,7 \%(\mathrm{n}=125.484)$ ocurrieron por enfermedades cardiovasculares, y de estas, $10 \%$ fueron registradas con diagnóstico primario de IC $(\mathrm{n}=12.825)$. Del total de egresos durante el año seleccionado, las hospitalizaciones por IC constituyeron el $0,98 \%$. Fueron eliminadas del análisis 231 hospitalizaciones, porque no contaban con la información del tipo de hospital, por lo que la muestra quedó constituida por 12.594 hospitalizaciones.

\section{Características epidemiológicas}

La Tabla 1 muestra la composición de la población en estudio. El 50\% de las hospitalizaciones ocurrieron en mujeres. La mediana de edad en mujeres fue mayor que en hombres, (76 años (67-83) vs 71 años (62-79); p < 0,001). El 88\% eran beneficiarios de FONASA, perteneciendo $86 \%$ de ellos a las categorías de menores ingresos (A/B). Respecto a los días de hospitalización, se observó tanto en hombres como en mujeres una mediana de 6 días. Cerca de 74\% de todos los egresos ocurrieron en centros de salud de alta complejidad (9.309), distribuidos de manera homogénea en hombres y mujeres ( $73,2 \%$ vs $74,6 \%$, respectivamente).

\section{Incidencia y prevalencia}

La tasa de incidencia de egresos por IC en población general fue de $0,71 \times 1.000$ habitantes para los hombres y $0,70 \times 1.000$ habitantes para las mujeres. En ambos grupos la mayor tasa de egresos se registró en los pacientes de 80 años y más $(11,89$ x 1.000 habitantes en hombres y 10,14 x 1.000 habitantes en mujeres) (Figura 1).

En la Figura 2 se muestra la prevalencia de egresos por IC en relación a los egresos por todas las causas (panel A) y a los egresos cardiovasculares (panel B). En el primero, se observa que las mujeres de 80 años y más representan el $4 \%$ del total de hospitalizaciones por IC, mientras que en los hombres el valor fue 3,3\%. En el panel $\mathrm{B}$, las hospitalizaciones por IC constituyeron aproximadamente $10 \%$ de las hospitalizaciones de causa cardiovascular; siendo más frecuentes en pacientes mujeres de 80 años y más $(19,1 \%$ vs $15,9 \% ; \mathrm{p}<0,001)$.

\section{Letalidad intrahospitalaria}

En la muestra total fue $9,2 \%$, siendo significativamente mayor en las mujeres en comparación a los hombres $(9,7 \%$ vs $8,6 \% ; p=0,029)$. El análisis

Tabla 1. Características de los egresos hospitalarios con diagnóstico primario de insuficiencia cardiaca en Chile según sexo, 2014

\begin{tabular}{|c|c|c|c|c|c|c|c|}
\hline \multirow{3}{*}{$\begin{array}{l}\text { n (\%) } \\
\text { Edad, años med (p25-75) }\end{array}$} & \multicolumn{2}{|c|}{ Total } & \multicolumn{2}{|c|}{ Hombre } & \multicolumn{2}{|c|}{ Mujer } & \multirow[t]{2}{*}{ p value } \\
\hline & \multicolumn{2}{|l|}{12.594} & 6.268 & 49,7 & 6.326 & 50,3 & \\
\hline & \multicolumn{2}{|c|}{$74(64-82)$} & \multicolumn{2}{|c|}{$71(62-79)$} & \multicolumn{2}{|c|}{$76(67-83)$} & $<0,001$ \\
\hline \multicolumn{8}{|l|}{ Previsión (\%) } \\
\hline FONASA A-B & 9.500 & 75,4 & 4.469 & 71,3 & 5.031 & 79,5 & $<0,001$ \\
\hline FONASA C-D & 1.603 & 12,7 & 947 & 17,1 & 656 & 10,3 & 0,001 \\
\hline ISAPRE $u$ otros & 1.491 & 11,8 & 852 & 13,5 & 639 & 10,1 & 0,041 \\
\hline Días estadía med (p25-75) & \multicolumn{2}{|c|}{$6(3-11)$} & \multicolumn{2}{|c|}{$6(3-10)$} & \multicolumn{2}{|c|}{$6(3-11)$} & NS \\
\hline \multicolumn{8}{|l|}{ Tipo de establecimiento (\%) } \\
\hline Baja complejidad & 3.285 & 26,1 & 1.681 & 26,8 & 1.604 & 25,3 & NS \\
\hline Alta complejidad & 9.309 & 73,9 & 4.587 & 73,1 & 4.722 & 74,6 & NS \\
\hline
\end{tabular}

*Para las variables continuas se presenta mediana y percentiles 25 y 75 . 


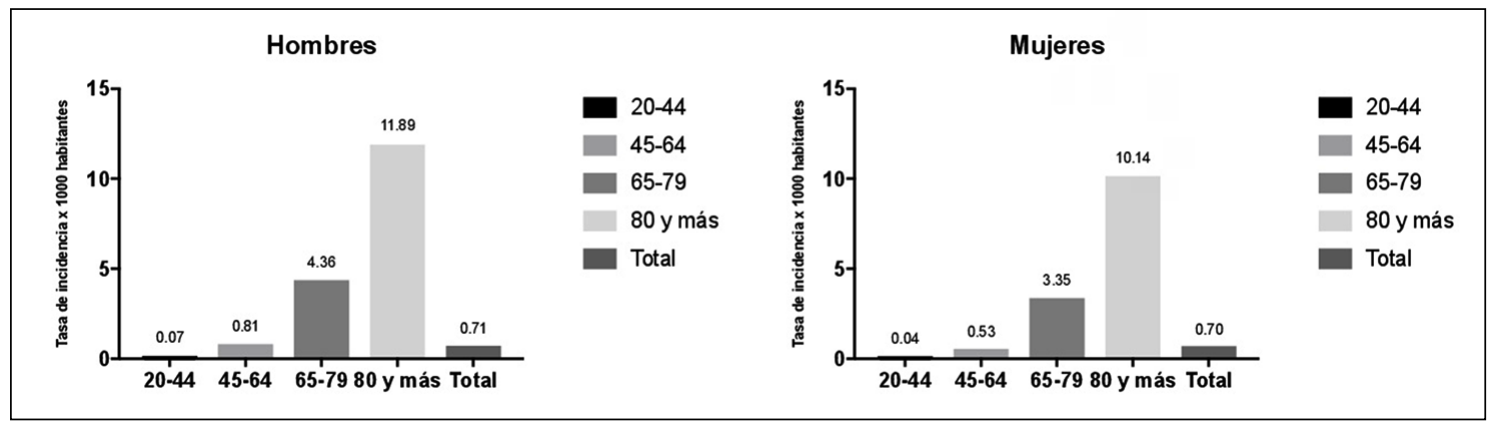

Figura 1. Tasa de incidencia de ingresos por insuficiencia cardiaca en población general según sexo, Chile 2014.

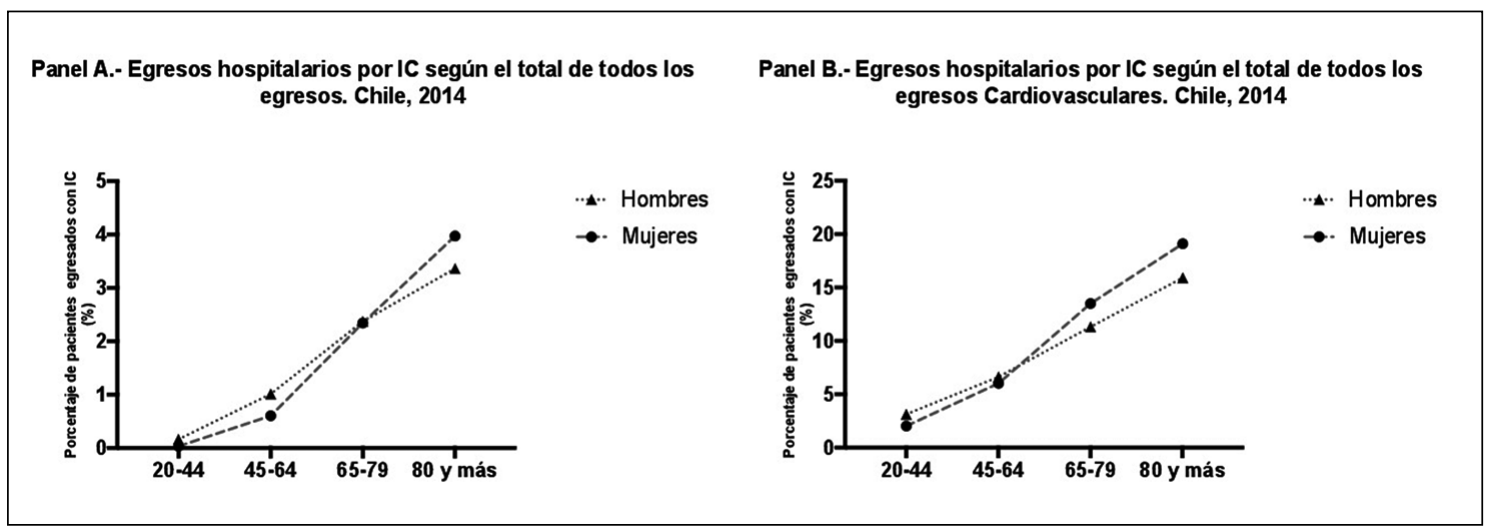

Figura 2. Proporciones de egresos hospitalarios por insuficiencia cardiaca, Chile 2014.

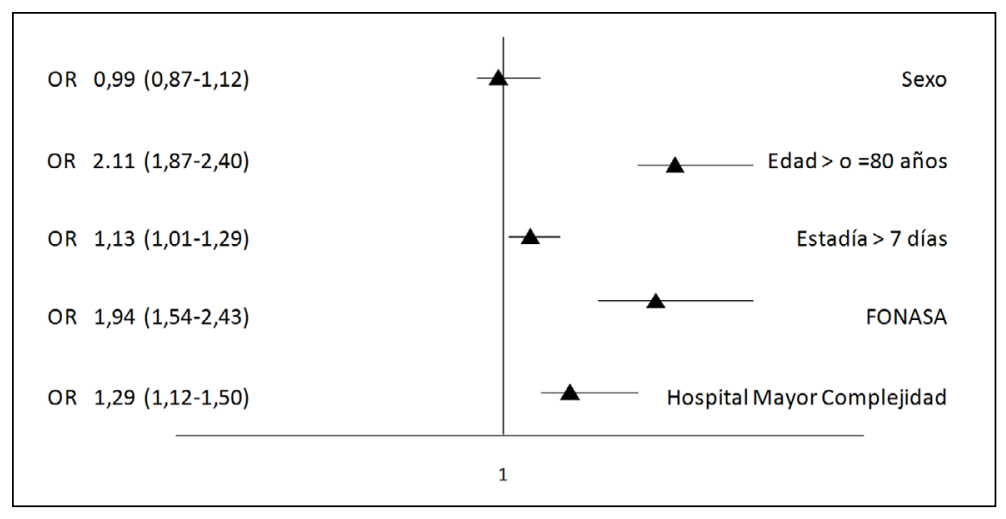

Figura 3. Factores asociados a letalitad intrahospitalaria por insuficiencia cardiaca, Chile 2014. Nota: OR= Odds Ratio, IC 95\%. crudo mostró que las mujeres presentaban 14\% más riesgo de morir durante la estadía hospitalaria (OR 1,14: IC 95\%; 1,01-1,29; p = 0,029). Sin embargo, en el análisis multivariado, el exceso de riesgo en las mujeres se anula al ajustar por edad (OR 0,96: IC 95\%; 0,85-1,10; $p=0,645)$. Los factores que se asociaron con mayor riesgo de muerte fueron: edad mayor a 80 años (OR 2,11; IC95\%: 1,87-2,40; $<<0,001)$, estadía hospitalaria superior a 7 días (OR 1,13; IC95\%: 1,01-1,29; $\mathrm{p}=0,04$ ), establecimientos de salud de alta complejidad (OR 1,29; IC95\%: 1,12-1,50; $\mathrm{p}=0,01$ ) y previsión FONASA (OR 1,94; IC95\%: 1,54-2,43; p < 0,001) (Figura 3). 


\section{Discusión}

Este trabajo entrega una primera aproximación de la morbimortalidad por IC en Chile empleando fuentes de datos nacionales. Estos resultados muestran diferencias, tanto en la incidencia como en la letalidad por IC, entre mujeres y hombres. Mientras que la incidencia de hospitalizaciones por IC es mayor en hombres y aumenta de manera significativa con la edad, por el contrario, la prevalencia de hospitalizaciones es más elevada en mujeres, quienes también presentan un mayor riesgo de muerte intrahospitalaria, efecto que desaparece al ajustar por edad. Los factores relacionados con mayor letalidad fueron: edad igual o mayor a 80 años, una estadía hospitalaria mayor a 7 días, hospitales de alta complejidad y estar afiliado a FONASA. Nuestros resultados muestran una prevalencia e incidencia menores a las reportadas en estudios internacionales ${ }^{1,16-19}$.

La relación del sexo con la epidemiología de la IC es controversial. Si bien muchos estudios poblacionales han mostrado que esta enfermedad afecta preferentemente a los hombres ${ }^{1,16,17}$, existe evidencia que la prevalencia e incidencia aumenta en mujeres a medida que estas envejecen. En el estudio Framingham ${ }^{17}$, las tasas de incidencia para hombres en comparación con las mujeres fueron 5,6 vs 3,3 por 1.000 personas años, respectivamente. Bui et al., en los Estados Unidos de Norteamérica, estimaron prevalencias mayores para hombres en todos los tramos de edad, alcanzando $14,7 \%$ para hombres y $12,7 \%$ para mujeres en el tramo etario sobre 79 años $^{16}$. Por el contrario, Mehta et al. reportaron hallazgos similares a los nuestros, con una prevalencia de IC en mujeres mayor que en hombres, incrementándose a medida que aumenta la edad $(7,9 \% \text { vs } 6,6 \% \text { respectivamente })^{18}$. También los estudios realizados en España, PRICE, BADAPIC, RAIC y ATTICA ${ }^{19,20}$, han mostrado cifras consistentes de prevalencias mayores en mujeres ( $7 \%$ vs $6,5 \%$, respectivamente, aunque en el caso de estudio BADAPIC se incluyeron pacientes de consulta ambulatoria).

Posibles explicaciones para esta aparente heterogeneidad radican en la dificultad que plantea el diagnóstico de IC en mujeres. En efecto, la forma más prevalente de IC en el sexo femenino de edad avanzada es aquella con fracción de eyección preservada $^{21}$, la cual frecuentemente genera dificultad diagnóstica.
De manera adicional, las mujeres desarrollan enfermedades cardiovasculares entre 7 y 10 años más tarde que los hombres, lo que explica la mayor prevalencia de IC en mujeres sobre 60 años ${ }^{22}$. También, el aumento en la expectativa de vida para las mujeres durante los últimos años excede al observado en los hombres a nivel mundial (73 años para las mujeres y 69 años para los varones), lo que se traduce en mayor sobrevida y, por ende, mayor probabilidad de desarrollar IC clínica en el último período de la vida ${ }^{23}$.

La letalidad intrahospitalaria global fue 9,2\%. En Latinoamérica y Estados Unidos de Norteamérica, diversos estudios han reportado cifras de letalidad intrahospitalaria entre $3 \%$ y $17 \%{ }^{1,6}$. En la región de las Américas se disponen de cifras en Brasil $^{24}$, donde es cercana a 7\%, y Argentina ${ }^{25}$, que muestra cifras que varían entre 4 y $12 \%$ según la localidad. En nuestro país, el registro nacional de Insuficiencia Cardiaca ICARO mostró que la estadía hospitalaria promedio fue de $10 \pm 9$ días y la mortalidad intrahospitalaria 5,6\% ${ }^{26}$.

Estas diferencias pueden deberse en parte a variaciones genéticas, calidad de la atención médica, complejidad de los servicios de salud, como también por las definiciones y métodos empleados para determinar la causa de muerte en los distintos países. Esto es de particular relevancia al considerar que al menos $20 \%$ de la mortalidad intrahospitalaria de pacientes admitidos por IC se atribuye primariamente a causas no cardiovasculares en Estados Unidos de Norteamérica ${ }^{1}$. Por tanto, la forma de registro de las bases clínicas y administrativas se traducen en una subestimación de la IC como diagnóstico de egreso y de muerte ${ }^{27-30}$.

En cuanto al efecto del sexo como predictor de muerte, en nuestro estudio no se encontró una asociación significativa después de ajustar por edad. Estos resultados son similares a otros reportados previamente por los estudios $\operatorname{ADHERE}^{13} \mathrm{y}$ OPTIMIZE- $\mathrm{HF}^{14}$, en donde la mortalidad intrahospitalaria no mostró diferencias entre ambos sexos. Similarmente, Hsich et al. mostraron que mujeres y hombres con fracción de eyección del ventrículo izquierdo reducida o preservada tienen similar letalidad intrahospitalaria ${ }^{21}$. Resultados similares se encuentran en el estudio de Parissis et al., concluyendo que la mortalidad intrahospitalaria por IC en hombres y mujeres no difiere significativamente $(10,5 \% \text { y } 11 \% \text { respectivamente })^{12}$.

La principal limitación de nuestro estudio es el 
empleo de datos provenientes de fuentes administrativas, los cuales son entregados por prestadores de salud y podrían no revelar los valores absolutos de las estimaciones que se presentan. Pese a esto, las bases de datos ministeriales siguen siendo, a nivel internacional, la herramienta más comúnmente empleada para la estimación de la magnitud de las enfermedades, en particular aquellas basadas en diagnósticos de egreso. En el caso de la IC, las estadísticas de mortalidad y egresos hospitalarios, utilizadas habitualmente para determinar la carga de enfermedad, subestiman la prevalencia, puesto que no siempre se reconoce como una entidad nosológica independiente de otra enfermedad del sistema cardiovascular ${ }^{26,27,31-34}$. Hay factores como la dificultad en el diagnóstico, la ausencia de marcadores específicos, la clasificación de la IC como mediador y no como causa de muerte, y la deficiente calidad de los registros hospitalarios que favorecen la subestimación de la IC como problema de salud ${ }^{35}$.

Una segunda limitación deriva del hecho que las estadísticas hospitalarias son basadas en eventos y no en sujetos, lo que permite que múltiples rehospitalizaciones de un único sujeto sean consideradas eventos diferentes, lo que limita la interpretación de las tasas de incidencia derivadas a partir de estos datos ${ }^{36}$. Si bien estas limitaciones metodológicas pueden afectar los estimados absolutos, nuestros resultados reflejan la distribución de la enfermedad y su letalidad por sexo resultando de utilidad para la cuantificación de la carga por IC.

\section{Conclusiones}

La IC es una patología prevalente dentro de las enfermedades cardiovasculares en Chile, cuya magnitud es difícil de estimar solamente a partir de fuentes de datos administrativos. Sin embargo, pese a las limitaciones, estos resultados muestran que el sexo femenino por sí sólo no constituye un factor asociado a mayor letalidad intrahospitalaria, sino que el exceso de riesgo observado se relaciona a su mayor edad. La edad, las estadías prolongadas, pertenecer a FONASA y ser admitido en un hospital de alta complejidad fueron factores que se asociaron a mayor riesgo de morir durante la hospitalización.

Este es uno de los primeros estudios en Chile que analiza la importancia de la IC en centros hospitalarios. Si bien el subdiagnóstico de la IC como entidad primaria de egreso en hospitales chilenos, se podría traducir en una menor estimación de la magnitud del problema, ante la ausencia de datos primarios, el análisis de la información basada en egresos hospitalarios es útil para conocer la distribución de la enfermedad en la población y favorecer los procesos de planificación de recursos públicos.

\section{Referencias}

1. Roger VL. Epidemiology of heart failure. Circ Res 2013; 113 (6): 646-59.

2. Blair JEA, Huffman M, Shah SJ. Heart failure in North America. Curr Cardiol Rev 2013; 9 (2): 128-46.

3. Heidenreich PA, Albert NM, Allen LA, Bluemke DA, Butler J, Fonarow GC, et al. Forecasting the Impact of Heart Failure in the United States. Circ Heart Fail 2013; 6 (3): 606-19.

4. van den Berge JC, Akkerhuis MK, Constantinescu AA, Kors JA, van Domburg RT, Deckers JW. Temporal trends in long-term mortality of patients with acute heart failure: Data from 1985-2008. Int J Cardiol 2016; 224: 456-60.

5. Bocchi E, Arias A, Verdejo H, Diez M, Gómez E, Castro P. The Reality of Heart Failure in Latin America. J Am Coll Cardiol 2013; 62 (11): 949-58.

6. Ciapponi A, Alcaraz A, Calderón M, Matta MG, Chaparro M, Soto N, et al. Burden of Heart Failure in Latin America: A Systematic Review and Meta-analysis. Rev Esp Cardiol (Engl Ed) 2016; 69 (11): 1051-60.

7. MacIntyre K, Capewell S, Stewart S, Chalmers JW, Boyd $\mathrm{J}$, Finlayson A, et al. Evidence of improving prognosis in heart failure: trends in case fatality in 66547 patients hospitalized between 1986 and 1995. Circulation 2000; 102 (10): 1126-31.

8. Benjamin EJ, Blaha MJ, Chiuve SE, Cushman M, Das SR, Deo R, et al. Heart Disease and Stroke Statistics-2017 Update: A Report From the American Heart Association. Circulation 2017; 135 (10): e146-e603.

9. Rich MW. Heart Failure in Women: Does Sex Matter? J Card Fail 2015; 21 (7): 539-40.

10. Kitzman DW, Gardin JM, Gottdiener JS, Arnold A, Boineau R, Aurigemma G, et al. Importance of heart failure with preserved systolic function in patients $>$ or $=65$ years of age. CHS Research Group. Cardiovascular Health Study. Am J Cardiol 2001; 87 (4): 413-9.

11. Nayar P, Yu F, Chandak A, Kan GL, Lowes B, Apenteng BA. Risk Factors for In-Hospital Mortality in Heart Failure Patients: Does Rurality, Payer or Admission Source Matter? J Rural Health 2016 Jun 8. doi: 10.1111/ jrh.12186. 
12. Parissis JT, Mantziari L, Kaldoglou N, Ikonomidis I, Nikolaou M, Mebazaa A, et al. Gender-related differences in patients with acute heart failure: management and predictors of in-hospital mortality. Int J Cardiol 2013; 168 (1): 185-9.

13. Galvao M, Kalman J, DeMarco T, Fonarow GC, Galvin C, Ghali JK, et al. Gender differences in in-hospital management and outcomes in patients with decompensated heart failure: analysis from the Acute Decompensated Heart Failure National Registry (ADHERE). J Card Fail 2006; 12 (2): 100-7.

14. Abraham WT, Fonarow GC, Albert NM, Stough WG, Gheorghiade M, Greenberg BH, et al. Predictors of in-hospital mortality in patients hospitalized for heart failure - Insights from the Organized Program to Initiate Lifesaving Treatment in Hospitalized Patients with Heart Failure (OPTIMIZE-HF). J Am Coll Cardiol 2008; 52 (5): 347-56.

15. Strömberg A, Mårtensson J. Gender differences in patients with heart failure. Eur J Cardiovasc Nurs 2003; 2 (1): 7-18.

16. Bui AL, Horwich TB, Fonarow GC. Epidemiology and risk profile of heart failure. Nat Rev Cardiol 2011; 8 (1): 30-41.

17. Ho KK, Pinsky JL, Kannel WB, Levy D. The epidemiology of heart failure: the Framingham Study. J Am Coll Cardiol 1993; 22 (4 Suppl A): 6A-13A.

18. Mehta PA, Cowie MR. Gender and heart failure: a population perspective. Heart 2006; 92 Suppl 3: iii14-8.

19. Anguita Sánchez M. Características clínicas, tratamiento y morbimortalidad a corto plazo de pacientes con insuficiencia cardíaca controlados en consultas específicas de insuficiencia cardíaca. Resultados del Registro BADAPIC. Rev Esp Cardiol 2004; 57 (12): 1159-69.

20. Jiménez-Navarro MF, Anguita Sánchez M. Insuficiencia cardiaca en la mujer. Diferencias de sexo en España. Rev Esp Cardiol Suplementos 2008; 8 (4): 23D-9.

21. Hsich EM, Grau-Sepúlveda MV, Hernández AF, Peterson ED, Schwamm LH, Bhatt DL, et al. Sex differences in in-hospital mortality in acute decompensated heart failure with reduced and preserved ejection fraction. Am Heart J 2012; 163 (3): 430-7, 437.e1-3.

22. Maas AH, Appelman YE. Gender differences in coronary heart disease. Neth Heart J 2010; 18 (12): 598-602.

23. Mendoza W, Miranda JJ. Global Shifts in Cardiovascular Disease, the Epidemiologic Transition, and Other Contributing Factors: Toward a New Practice of Global Health Cardiology. Cardiol Clin 2017; 35 (1): 1-12.

24. Bocchi EA, Vilas-Boas F, Perrone S, Caamaño AG, Clausell N, Moreira MDCV, et al. I Latin American Guidelines for the Assessment and Management of
Decompensated Heart Failure. Arq Bras Cardiol 2005; 85 Suppl 3: 49-94; 1-48.

25. Perna ER, Barbagelata A, Grinfeld L, García Ben M, Címbaro Canella JP, Bayol PA, et al. Overview of acute decompensated heart failure in Argentina: lessons learned from 5 registries during the last decade. Am Heart J 2006; 151 (1): 84-91.

26. Castro P, Verdejo H, Vukasovic R, Garcés E, González I. Predictores de mortalidad intrahospitalaria y hospitalización prolongada en la insuficiencia cardíaca: resultados preliminares del registro nacional de insuficiencia cardíaca. Grupo ICARO. Rev Med Chile 2006; 134 (9): 1083-91.

27. Snyder ML, Love S-A, Sorlie PD, Rosamond WD, Antini C, Metcalf PA, et al. Redistribution of heart failure as the cause of death: the Atherosclerosis Risk in Communities Study. Popul Health Metr 2014; 12 (1): 10.

28. Khand AU, Shaw M, Gemmel I, Cleland JGF. Do discharge codes underestimate hospitalisation due to heart failure? Validation study of hospital discharge coding for heart failure. Eur J Heart Fail 2005; 7 (5): 792-7.

29. Ingelsson E, Arnlöv J, Sundström J, Lind L. The validity of a diagnosis of heart failure in a hospital discharge register. Eur J Heart Fail 2005; 7 (5): 787-91.

30. Kümler T, Gislason GH, Kirk V, Bay M, Nielsen OW, Køber L, et al. Accuracy of a heart failure diagnosis in administrative registers. Eur J Heart Fail 2008; 10 (7): 658-60.

31. Murray CJL, Kulkarni SC, Ezzati M. Understanding the coronary heart disease versus total cardiovascular mortality paradox: a method to enhance the comparability of cardiovascular death statistics in the United States. Circulation 2006; 113 (17): 2071-81.

32. Murdoc D. Underestimation of heart failure as a cause of death. Cardiology Rev 2000; 17 (6): 13-6.

33. Antini C, Rajs D, Muñoz-Quezada MT, Mondaca BAL, Heiss G. Reliability of cause of death coding: an international comparison. Cad Saude Publica 2015; 31 (7): 1473-82.

34. Mähönen M, Jula A, Harald K, Antikainen R, Tuomilehto J, Zeller $\mathrm{T}$, et al. The validity of heart failure diagnoses obtained from administrative registers. European Journal of Preventive Cardiology. Eur J Prev Cardiol 2013; 20 (2): 254-9.

35. Fonseca C, Sarmento PM, Marques F, Ceia F. Validity of a discharge diagnosis of heart failure: implications of misdiagnosing. Congest Heart Fail 2008; 14 (4): 187-91.

36. McCormick N, Lacaille D, Bhole V, Avina-Zubieta JA. Validity of heart failure diagnoses in administrative databases: a systematic review and meta-analysis. PLoS One 2014; 9 (8): e104519. 